06,11

\title{
Особенности структуры промежуточной фазы в цирконате-титанате свинца с высоким содержанием циркония
}

\author{
(ㄱ Д.А. Андроникова ${ }^{1}$, Ю.А. Бронвальд ${ }^{1,2}$, Н.Г. Леонтьев ${ }^{3}$, И.Н. Леонтьев ${ }^{4}$, Д.Ю. Чернышов ${ }^{2,5}$, \\ А.В. Филимонов ${ }^{2}$, С.Б. Вахрушев ${ }^{1}$ \\ ${ }^{1}$ Физико-технический институт им. А.Ф. Иоффре РАН, \\ Санкт-Петербург, Россия \\ ${ }^{2}$ Санкт-Петербургский политехнический университет Петра Великого, \\ Санкт-Петербург, Россия \\ ${ }^{3}$ Азово-Черноморский инженерный институт ФГБОУ ВО Донской ГАУ, \\ Зерноград, Россия \\ ${ }^{4}$ Южный федеральный университет, \\ Ростов-на-Дону, Россия \\ ${ }^{5}$ Swiss-Norwegian Beamlines at ESRF, \\ Гренобль, Франция \\ E-mail: andronikova.daria@gmail.com \\ Поступила в Редакцию 27 мая 2019 г. \\ В окончательной редакции 27 мая 2019 г. \\ Принята к публикации 29 мая 2019 г.
}

\begin{abstract}
Приводятся результаты эксперимента по дифракции рентгеновского излучения на монокристалле цирконата-титаната свинца $\mathrm{PbZr}_{0.993} \mathrm{Ti}_{0.007} \mathrm{O}_{3}$ в области существования промежуточной сегнетоэлектрической фазы. Дополнительно к известным ранее сверхструктурным рефлексам М-типа $q_{M}=\left\{\frac{1}{2}, \frac{1}{2}, 0\right\}$ и сателлитным отражениям первого порядка $q_{M}+\{\delta, \delta, \delta\}$ обнаружены ранее неизвестные сателлиты второго порядка в окрестности $q_{M}$ и вблизи фундаментальных брэгговских пиков. Построена модель, предполагающая существование регулярной системы антифазных доменов. Показано, что данная модель описывает сателлитные отражения первого и второго порядков в окрестности $q_{M}$, но не может объяснить появление сателлитов вокруг основных брэгговских пиков. Обсуждается возможная природа наблюдаемой картины сверхструктурных отражений в промежуточной фазе.
\end{abstract}

Ключевые слова: сегнетоэлектрики, фазовые переходы, рассеяние рентгеновского излучения, структура перовскита,кристаллическая структура.

DOI: 10.21883/FTT.2019.10.48254.494

\section{1. Введение}

Цирконат-титанат свинца $\mathrm{PbZr}_{1-x} \mathrm{Ti}_{x} \mathrm{O}_{3}$ (ЦТС) - активно исследуемый перовскитоподобный функциональный материал, проявляющий как сегнетоэлектрические, так и антисегнетоэлектрические свойства в зависимости от концентрации титана. Сегнетоэлектрический ЦТС (PZT) популярен среди исследователей благодаря высоким пьезоэлектрическим коэффициентам, делающим его одним из лидеров на рынке пьезоэлектрических материалов [1]. Антисегнетоэлектрический ЦТС вызывает интерес как модельный объект для исследования природы антисегнетоэлектрического состояния, до сих пор являющейся поводом для дискуссий [2-5]. Также стоит отметить все возрастающий интерес к антисегнетоэлектрическим материалам как к перспективным материалам для создания систем охлаждения, основанных на электрокалорическом эффекте [6,7], устройств накопления энергии конденсаторного типа [8], а также устройств хранения информации высокой плотности записи $[9,10]$.
В области концентраций $0<x<0.04$ [11] ЦТС испытывает два фазовых перехода: из кубической параэлектрической фазы в промежуточную и далее в антисегнетоэлектрическую фазу. В то время как структура антисегнетоэлектрической и параэлектрической фаз являются достаточно хорошо изученной [12-16], структура промежуточной фазы, реализующейся между ними, до сих пор остается открытым вопросом. Известно, что данная фаза является нецентросимметричной [17]. В работе [16] наблюдались сегнетоэлектрические петли гистерезиса. Было предположено, что ЦТС в промежуточной фазе имеет ромбоэдрическую структуру [16] с пространственной группой $R 3 m[17]$.

Фазовый переход из параэлектрической в промежуточную фазу характеризуется возникновением сверхструктурных рефлексов в точках $\left\{\frac{1}{2}+h, \frac{1}{2}+k, l\right\}[13,18,19]$, где $h, k, l$ - индексы Миллера в псевдокубической индексации. В дальнейшем данные отражения мы будем называть сверхструктурными рефлексами М-типа. Возникновение сверхструктурных рефлексов М-типа является следствием удвоения элементарной ячейки кубической фазы в двух направлениях. В работе [16] возникновение 
данных сверхструктур связали с конденсацией моды $M_{3}$, которая приводит к софазным разворотам кислородных октаэдров (в соседних слоях октаэдры разворачиваются в одном направлении). Однако в работе [20] было отмечено, что в дифракционных картинах не наблюдаются систематические погасания, характерные для дифракции на структуре перовскита с малыми поворотами октаэдрических групп. Был предположен иной механизм возникновения данных сверхструктур - антипараллельные смещения ионов свинца, соответствующие конденсации $M_{5}$ моды.

Описание структуры промежуточной фазы осложняется существованием сателлитных рефлексов в окрестности сверхструктурных отражений М-типа [19-23]. В работе [20] сателлитные рефлексы не наблюдались в монокристаллических образцах, вследствие чего авторы предположили, что сателлитные рефлексы - особенность керамических образцов и являются результатом рассеяния на поверхности. На основании измерений темнопольной электронной микроскопии [20] данные сателлитные рефлексы были соотнесены с регулярной системой антифазных доменных границ. Период данных доменов соответствует обратному расстоянию между сателлитными рефлексами. В работах $[21,22]$ было предложено, что, помимо антифазных доменных границ, в сателлитные рефлексы вносит вклад другой тип доменов, предположительно соответствующих различным ориентациям поворотов кислородных октаэдров в $M_{3}$ моде. Сегнетоэлектрические свойства промежуточной фазы в работе [21] были объяснены существованием сегнетоэлектрических доменов, поляризация в которых направлена вдоль $\langle 100\rangle$ направлений.

Как следствие, в литературе встречается несколько вариантов объяснения природы данных сателлитов. В работе [19] сателлитные рефлексы связывают с модуляцией угла вращения кислородных октаэдров в моде $M_{3}$. Авторы работы [20] связывают возникновение сателлитов с рассеянием на антифазных доменах, внутри которых ионы свинца смещены антипараллельно. Под антифазными доменами понимаются домены, имеющие одну и ту же кристаллическую структуру и макроскопические свойства, но разделенные антифазными доменными границами, в которых направление смещений меняется на противоположное [24]. В работе [22] сателлитные рефлексы были интерпретированы как результат рассеяния на комбинации смещений различной симметрии: антипараллельных антисегнетоэлектрических смещений, соответствующих моде $M_{5}$, и кислородных смещений, соответствующих моде $M_{3}$.

Отметим, что вся обсуждаемая в литературе информация о системе сателлитных рефлексов в ЦТС получена методом электронной дифракции в керамических образцах. В данной статье приведены результаты исследования промежуточной фазы методом дифракции синхротронного излучения на монокристаллическом ЦТС. Использование высокоинтенсивного излучения и позиционно-чувствительного детектора широкого динамиче- ского диапазона позволило получить принципиально новую информацию о структуре промежуточной фазы. Были проведены количественные расчеты дифракционной картины в рамках модели, учитывающей существование регулярной структуры антифазных доменов. Показано, что в рамках данной модели удается получить хорошее согласие относительных интенсивностей и положений сателлитных рефлексов первого и второго порядков в окрестности сверхструктурных рефлексов М-типа, но невозможно воспроизвести впервые обнаруженные сателлитные рефлексы в окрестности брэгговских рефлексов.

\section{2. Дифракционные измерения}

\section{1. Характеристика образцов и описание эксперимента}

Монокристалл $\mathrm{PbZr}_{0.993} \mathrm{Ti}_{0.007} \mathrm{O}_{3}$ (ЦТС0.7) был выращен по технологии, описанной в работе [11]. Выращенные образцы имели форму параллелепипеда с размерами около $100 \times 100 \times 1500(\mu \mathrm{m})$. Подготовка образцов к дифрактометрическим измерениям включала в себя стачивание до размеров, обеспечивающих минимальное поглощение синхротронного излучения, и последующее травление в соляной кислоте. В результате образец имел размеры $50 \times 50 \times 500(\mu \mathrm{m})$.

Измерения проводились на универсальном дифрактометре PILATUS SNBL швейцарско-норвежской линии синхротронного источника ESRF. Монокристалл с помощью высокотемпературного керамического клея крепился к кварцевому капилляру, который фиксировался в гониометре. Регистрация рассеянного излучения осуществлялась с применением позиционно-чувствительного детектора PILATUS 2M [25]. Сканирование обратного пространства производилось путем вращения образца относительно вертикальной оси, шаг вращения составлял $0.25^{\circ}$, экспозиция на каждом шаге - $1 \mathrm{~s}$. Длина волны падающего излучения составляла $0.95 \AA$. Температура на образце регулировалась посредством обдува образца струей азота заданной температуры с точностью поддержания температуры в $0.5^{\circ}$.

В работе [26] на основании диэлектрических и оптических исследований фазовых переходов в аналогичных монокристаллах ЦТС была получена уточненная фазовая диаграмма в диапазоне концентраций $0<x<0.05$. Согласно полученным результатам промежуточная фаза в ЦТС 0.7 в режиме охлаждения наблюдается в диапазоне температур 494-455 K, при нагреве - 487.5-507 К. Результаты, описанные в настоящей работе, были получены при температуре 493 К с выходом на эту температуру в режиме нагрева, что соответствует промежуточной сегнетоэлектрической фазе.

Обработка полученных дифракционных картин, включающая в себя индексацию рефлексов и нахождение матрицы ориентации, была проведена с использованием программы CrysAlisPro [27]. Индексация рефлексов в 


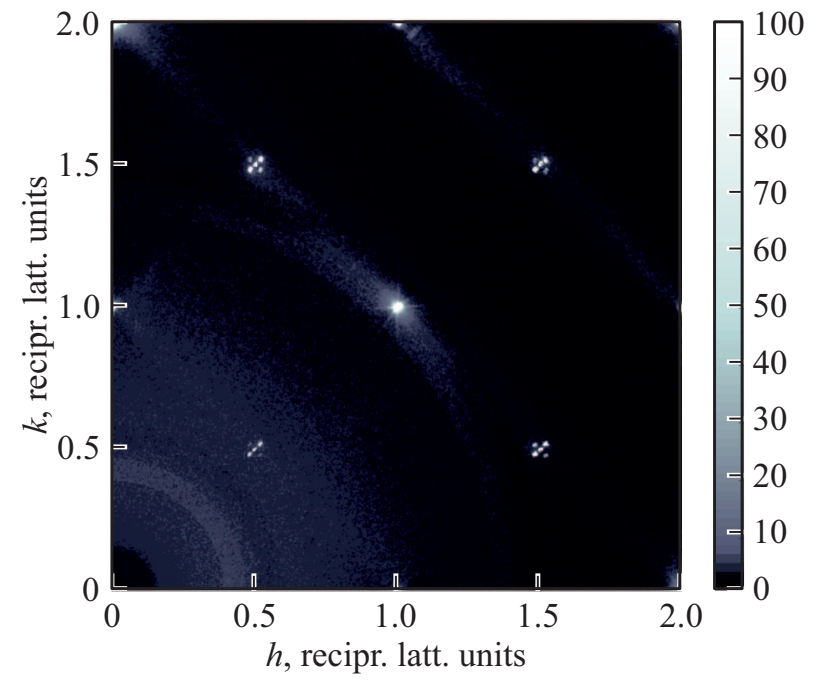

Рис. 1. Сечение обратного пространства плоскостью $(h k 0)$ в монокристалле ЦТС0.7 в промежуточной фазе. Интенсивность проинтегрирована в диапазоне $-0.02<l<0.02$ r.l.u. Цветовая шкала отображает значения интенсивности в относительных единицах.

промежуточной фазе выполнена в псевдокубических координатах. Реконструкция обратного пространства, в результате которой было получено трехмерное распределение интенсивности рассеянного излучения в координатах обратного пространства, осуществлялась с помощью пакета программ, написанных в среде MATLAB.

\section{2. Система сверхструктурных отражений}

На рис. 1 приведено сечение обратного пространства плоскостью $(h k 0)$ в промежуточной фазе ЦТС0.7. Картина рассеяния характеризуется наличием сверхструктурных отражений М-типа в точках $\left\{\frac{1}{2}+h, \frac{1}{2}+k, l\right\}$, a также дополнительных сателлитных рефлексов в их окрестности. Сверхструктурные рефлексы наблюдаются при всех значениях $h, k$ и $l$, в том числе и при $h=k$. Следовательно, для сверхструктурных рефлексов М-типа отсутствуют систематические погасания, характерные при рассеянии на каркасе кислородных октаэдров, испытывающих софазные развороты [28]. Это согласуется с наблюдениями, сделанными в работе [20], и служит основанием для предположения, что возникновение сверхструктурных рефлексов М-типа связано с искажениями кубической решетки, включающими катионные смещения.

Построены трехмерные распределения интенсивности синхротронного излучения в окрестности основных и сверхструктурных рефлексов М-типа. На рис. 2 приведены такие картины трехмерного распределения интенсивности в координатах обратного пространства вблизи $Q_{M}=(0,0.5,1.5)$ и $Q_{B r}=(0,0,2)$. Согласно полученным распределениям в окрестности сверхструктуры М-типа наблюдается 8 сателлитных рефлексов, равноудаленных от $Q_{M}$ узла и смещенных в направлениях $\langle 111\rangle$. Впервые установлено, что в некоторых направлениях наблюдаются сателлиты второго порядка, находящиеся на удвоенном расстоянии от $Q_{M}$ по сравнению с сателлитами первого порядка. Картина распределения сателлитов схожа для всех отражений М-типа: в окрестности любого из них существуют сателлитные рефлексы как первого, так и второго порядков. Однако направления, в которых наблюдаются сателлиты второго порядка, различаются для сверхструктурных рефлексов с различными индексами Миллера.

Важным новым результатом является наблюдение существования сателлитных рефлексов в окрестности основных узлов обратной решетки (см. рис. 2,b) Насколько нам известно, это первое сообщение о сателлитах в окрестности основных узлов в ЦТС: в работах $[20,22,23]$ упоминания о существовании сателлитов вокруг брэгговских узлов отсутствуют. Данные сателлиты смещены относительно брэгговского рефлекса в направлениях $\langle 111\rangle$. Учитывая расстояние между брэгговским рефлексом и сателлитами, можно утверждать, что данные сателлиты являются сателлитами второго порядка. Направления, в которых наблюдаются сателлиты в окрестности брэгговских рефлексов, совпадают с направлениями, в которых наблюдаются сателлиты второго порядка вблизи сверхструктур М-типа.

\section{3. Моделирование картин рассеяния от регулярной структуры антифазных доменов}

\section{1. Описание модели}

Как известно из результатов электронной микроскопии $[20,21,23]$, в ЦТС можно ожидать существования регулярной системы антифазных доменов. Мы провели расчеты дифракционных картин в рамках модели, учитывающей существование такой регулярной структуры. Моделирование картины рассеяния от системы антифазных доменов состояло из двух этапов: задание положения всех атомов кристаллической решетки в прямом пространстве и расчет интенсивности рассеяния от данной структуры в различных точках обратного пространства. Все расчеты проведены с использованием среды программирования MATLAB.

В параэлектрической фазе ЦТС имеет структуру кубического перовскита $A B O_{3}$, где атом $A$ находится в углах элементарной ячейки, атом $B-$ в ее центре, атомы кислорода — в центрах граней. В рамках используемой модели предполагалось, что возникающие сверхструктурные рефлексы могут быть полностью соотнесены со смещениями ионов свинца. Это предположение основывается на высокой интенсивности рассеяния рентгеновского излучения и отсутствия систематических погасаний сверхструктурных рефлексов 

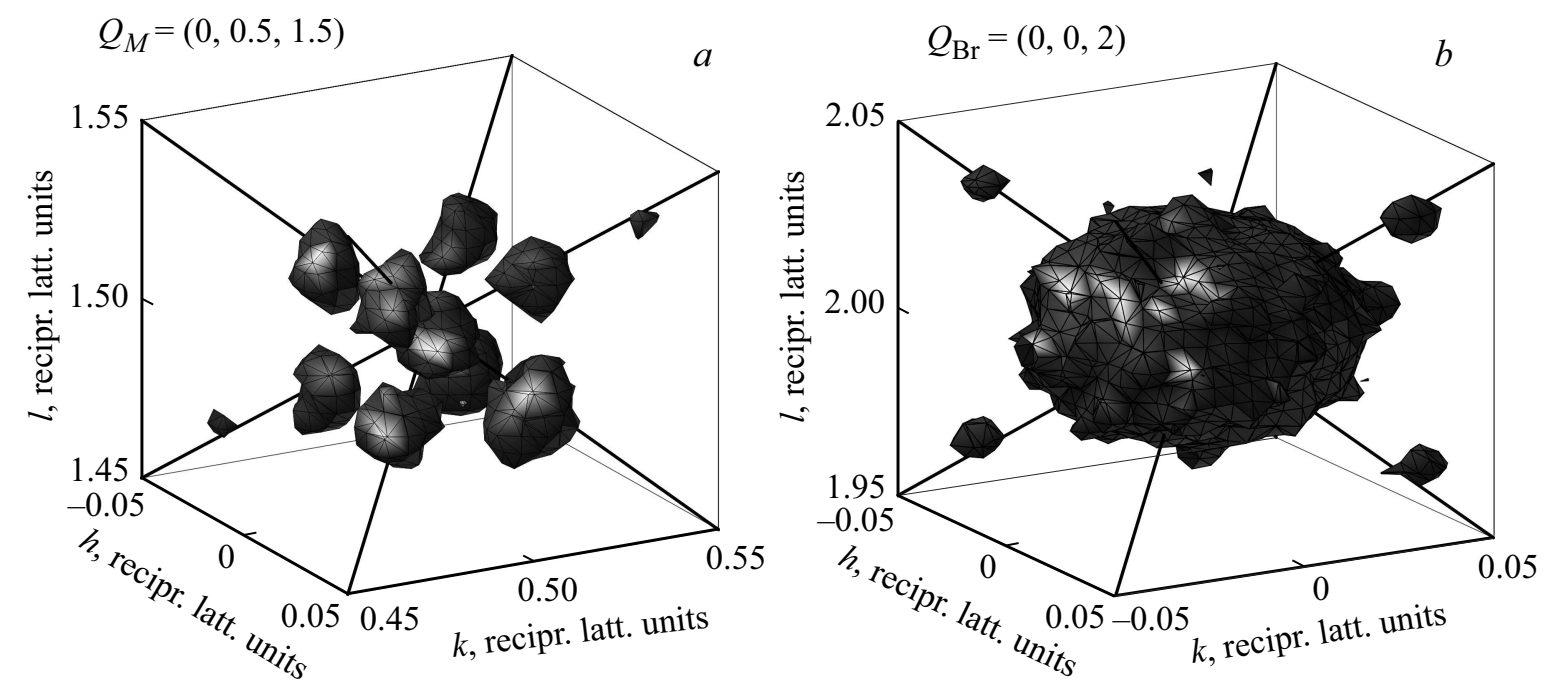

Рис. 2. Трехмерное распределение интенсивности синхротронного излучения в окрестности: а) сверхструктурного рефлекса М-типа, $Q_{M}=(0,0.5,1.5)$ и b) в окрестности брэгговского рефлекса $Q_{B r}=(0,0,2)$ в промежуточной фазе $(T=473 \mathrm{~K})$. Линиями указаны направления типа $\langle 111\rangle$.
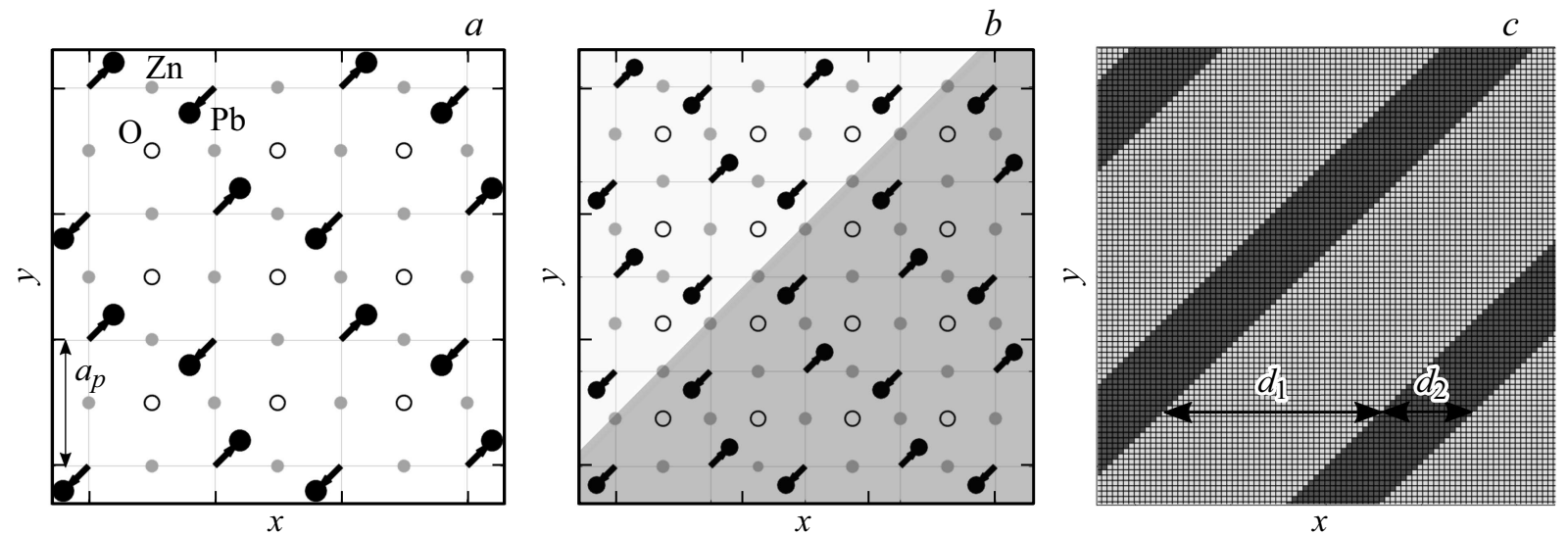

Рис. 3. Схематичное представление расположения атомов в рассматриваемой модели в $(x y 0)$ плоскости а) внутри одного домена, b) в окрестности антифазной доменной границы. Серые точки - атомы кислорода, белые круги - атомы циркония, черные круги - атомы свинца, стрелки указывают направления смещений атомов свинца. На рисунках показана проекция положений атомов $\mathrm{Zr}$ и О на плоскость, в которой происходят смещения ионов свинца. Обозначенные атомы циркония и кислорода располагаются на $a_{p} / 2$ выше. с) Система регулярных антифазных доменов, разделенных доменными границами.

М-типа. Таким образом, только атомы свинца в данной модели имели положения, отличные от их положений в структуре кубического перовскита. Координаты ионов свинца задавались в соответствии с предложенной в работе [20] схемой, согласно которой атомы свинца

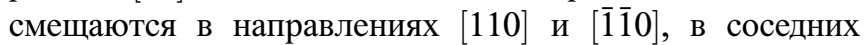
ячейках смещения разнонаправленны (см. рис. 3, $a$ ). Величина смещений полагалась равной $0.1 a_{p}$, где $a_{p}-$ постоянная псевдокубической ячейки.

Наличие в соседних ячейках антипараллельных смещений приводит к удвоению периода в двух направлениях, и, как следствие, к возникновению сверхструктурных рефлексов М-типа. Для возникновения сателлитов необходимо существование дополнительной мезоструктуры, характеризующейся периодом в несколько десятков эле- ментарных ячеек. В работах $[20,22]$ предполагалось, что такая мезоструктура может быть создана за счет антифазных доменов, разделенных антифазными доменными границами. Толщина антифазных доменных границ в общем случае может составлять несколько элементарных ячеек [9]. В используемой модели толщина антифазных доменных границ была задана равной $2 a_{p}$ (см. рис. $3, b$ ).

Из анализа картин рассеяния известно, что сателлитные рефлексы смещены относительно $q_{M}$ в $\langle 111\rangle$ направлениях. Можно предположить, что плоскости антифазных доменных границ должны иметь наклон относительно оси $z$ и быть ориентированы по нормали к $\langle 111\rangle$. При расчетах нормаль к плоскостям домен-

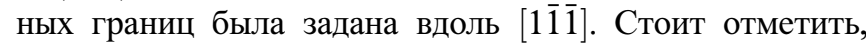
что наклон антифазных доменов относительно оси $z$ 


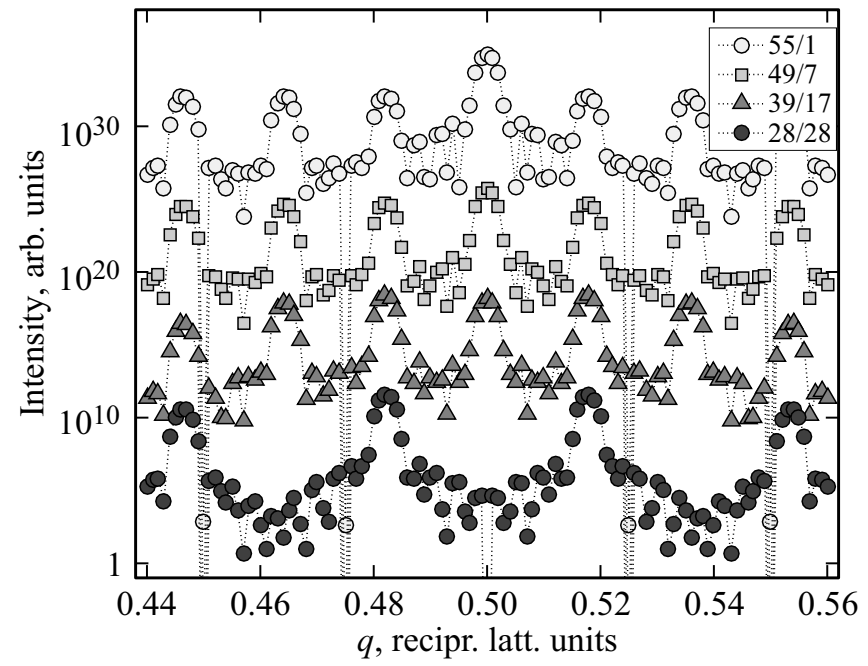

Рис. 4. Распределение интенсивности в окрестности $Q_{M}=(1.5,0.5,0)$ вдоль $[1.5+q, 0.5-q, q]$ линии, рассчитанное при различных отношениях $d_{1} / d_{2}: 28 / 28,39 / 17,49 / 7$ и $55 / 1$.

приводит к тому, что в соседних $(x y 0)$ плоскостях направления смещений атомов свинца в антифазной доменной границе, и, как следствие, поляризация, имеют противоположные направления.

Таким образом, полученная мезоструктура может быть представлена в виде системы регулярных антифазных доменов, разделенных доменными границами.

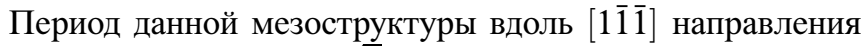
можно записать как $\sqrt{3} d$, где $d=d_{1}+d_{2}$ (см. обозначения на рис. $3, c$ ). Расстояние $d$ между плоскостями антифазных доменных границ однозначно соотносится с расстоянием между сателлитами и сверхструктурным рефлексом М-типа. Из дифракционных данных было установлено, что положение сателлитных рефлексов первого порядка может быть описано как $q_{M}+\{\delta, \delta, \delta\}$, где $\delta \sim 0.018$ r.l.u. Таким образом, $d=1 / \delta \sim 56 a_{p}$.

Для простоты расчетов моделирование было проведено для цирконата свинца, то есть в пренебрежении $0.7 \%$ включениями титана. Мы предполагаем, что данное упрощение не должно привести к существенному искажению результатов, так как, во-первых, атомы циркония и титана не участвуют в формировании рассматриваемой мезоструктуры. Во-вторых, в цирконате свинца промежуточная фаза также характеризуется наличием сателлитных рефлексов в окрестности брэгговских рефлексов и сверхструктур М-типа [29]. Следовательно, добавление $0.7 \%$ титана качественным образом не меняет картину рассеяния в промежуточной фазе.

Интенсивность рассеяния рассчитывалась как квадрат структурного фактора

$$
I(Q) \propto F(Q)^{2}=\left(\sum_{c} \sum_{m} f_{m} \exp \left(-i 2 \pi r_{m} Q\right)\right)^{2}
$$

где $f_{m}-$ атомный фактор атома $m$, значения $f_{m}$ взяты из [30], $r_{m}$ - положение атома $m, Q-$ переданный волновой вектор. Суммирование ведется по всем атомам в элементарной ячейке $(m)$ и по всем ячейкам $(c)$. При вычислении интенсивности не учитывался параметр Дебая-Уоллера. Очевидно, что такой расчет не позволяет воспроизвести точное соотношение относительной интенсивности основных и сателлитных рефлексов. Тем не менее, в рамках данной модели мы имеем возможность воспроизвести ориентацию и положение сателлитных рефлексов.

При вычислении трехмерных распределений интенсивности расчеты велись на системе из $112 \times 112 \times 112$ элементарных ячеек $a_{p}$, включающей 13 антифазных доменов. Расчеты одномерных профилей интенсивности вдоль $\langle 111\rangle$ направлений были проведены для объема, включающего $280 \times 280 \times 200$ элементарных ячеек. Расчеты выполнялись с шагом 0.002 r.l.u. для трехмерных распределений интенсивности и с шагом 0.001 r.l.u. для одномерных.

\section{2. Результаты расчета}

Как было сказано ранее, из анализа дифракционных картин может быть определен период $d$ рассматриваемой мезоструктуры, при этом отношение $d_{1} / d_{2}$ является варьируемым параметром. Было рассмотрено несколько различных вариантов отношения величин $d_{1}$ и $d_{2}$. На рис. 4 показаны одномерные профили распределения интенсивности в направлении [11ㅣ] в окрестности сверхструктурного рефлекса М-типа, рассчитанные при различных значениях $d_{1} / d_{2}$. Расчеты показали, что от отношения ширин доменов значительно зависит отношение интенсивностей сателлитов и сверхструктурного рефлекса М-типа. Стоит отметить, что при рассеянии на доменах одной ширины непосредственно в М точке рефлекс не наблюдается.

Для дальнейших вычислений было выбрано соотношение $d_{1} / d_{2}=49 / 7$ как дающее наиболее близкую картину рассеяния к экспериментально наблюдаемой. На рис.5, $а$ приведено сравнение рассчитанного и полученного экспериментально одномерного распределения интенсивности в направлении [11ㅣ вблизи $Q_{M}=(1.5,0.5,0)$. Полученная картина характеризуется наличием рефлекса непосредственно в точке $Q_{M}$, а также набором сателлитов различных порядков, интенсивность которых спадает с увеличением порядка. Наблюдается хорошее совпадение положений сателлитных рефлексов относительно сверхструктуры М-типа, а также относительных интенсивностей сателлитных рефлексов первого порядка и сверхструктурного рефлекса.

На рис. 5, $b$ приведены результаты расчета интенсивности в небольшом объеме обратного пространства в окрестности $Q_{M}=(1.5,0.5,0)$. В направлении $[1 \overline{1} 1]$ наблюдается цепочка сателлитных рефлексов. Данная картина согласуется с экспериментально наблюдаемой, если предположить, что цепочка сателлитов в одном из 

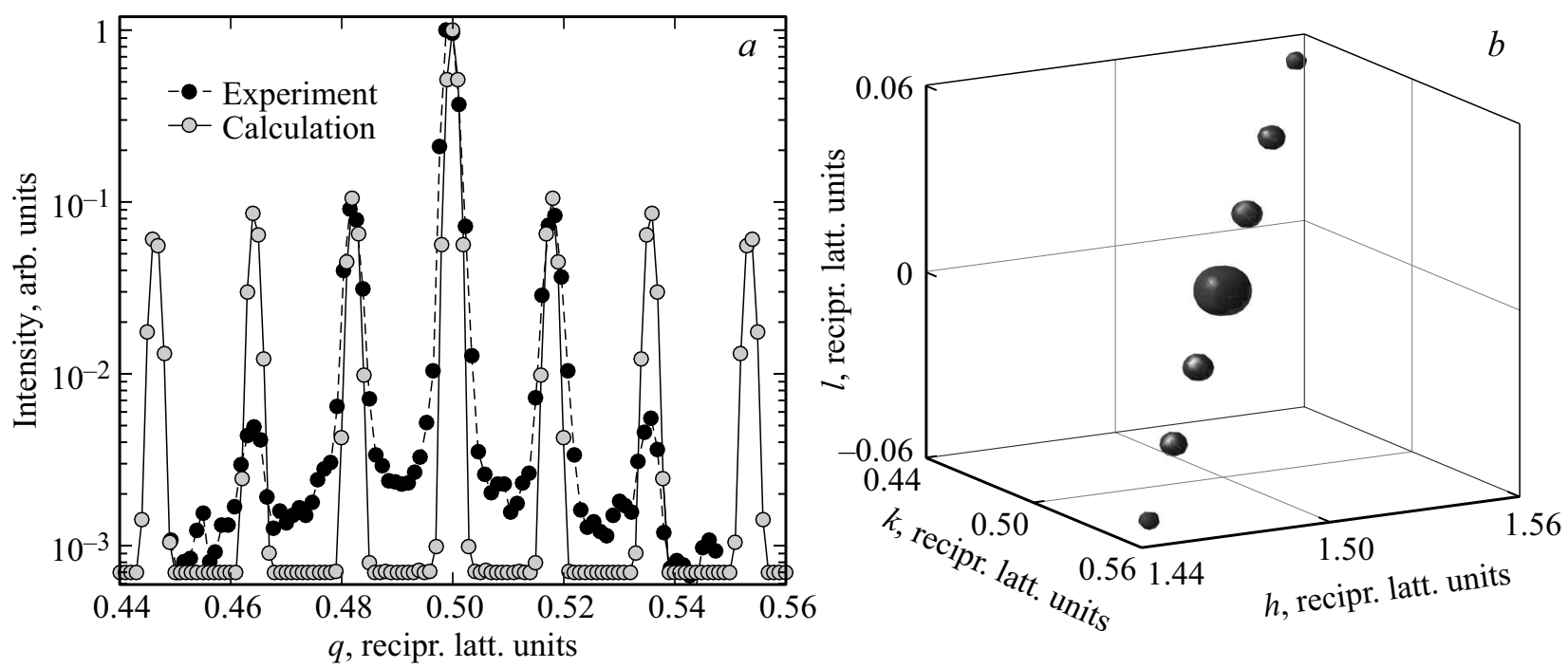

Рис. 5. а) Сравнение рассчитанного (серые точки) и экспериментально полученного (черные точки) профилей интенсивности в окрестности $Q_{M}=(1.5,0.5,0)$ вдоль [111] направления. Интенсивности нормированы на максимальное значение. b) Рассчитанное трехмерное распределение интенсивности в окрестности $Q_{M}=(1.5,0.5,0)$. При расчетах отношение $d_{1} / d_{2}$ составляло $49 / 7$.
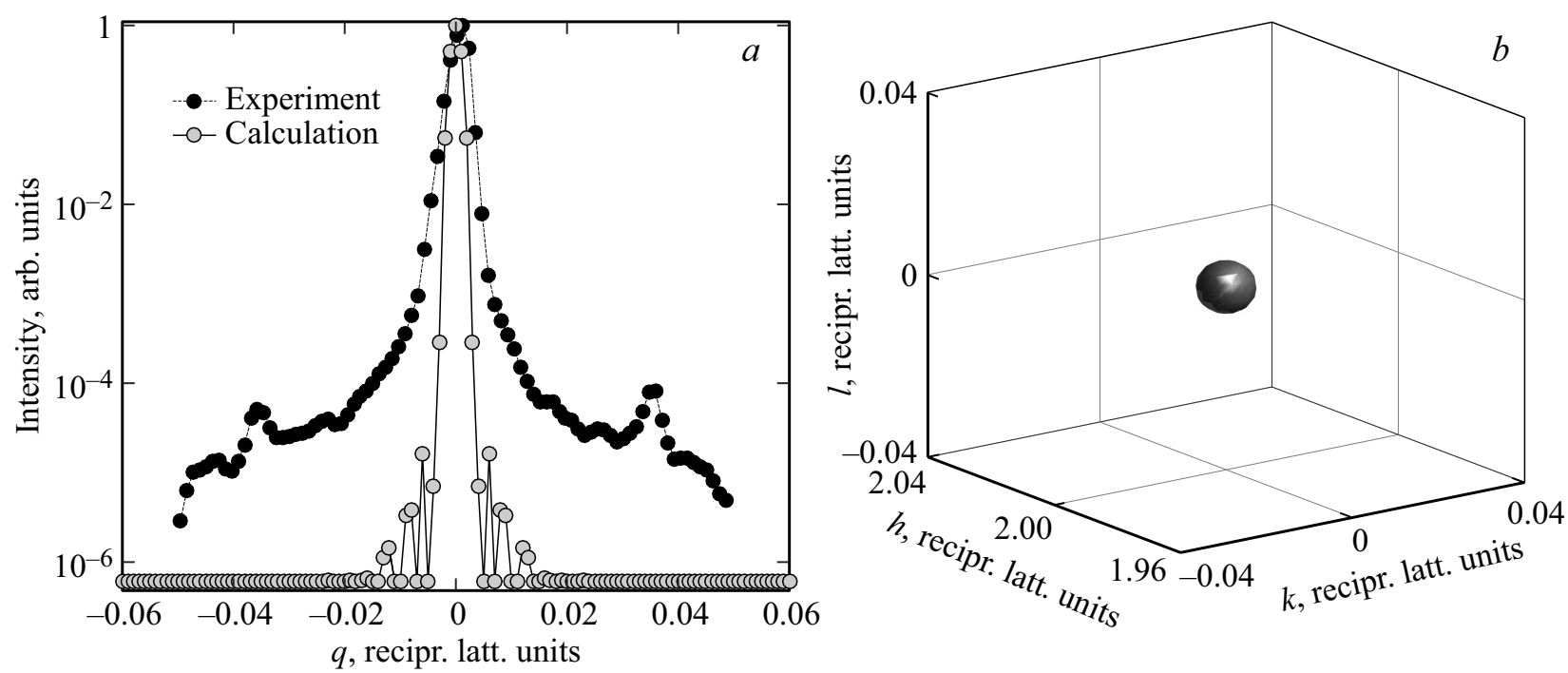

Рис. 6. а) Сравнение рассчитанного (серые точки) и экспериментально полученного (черные точки) профилей интенсивности в окрестности $Q_{B r}=(2,0,0)$ вдоль [1111] направления. Интенсивности нормированы на максимальное значение. b) Рассчитанное трехмерное распределение интенсивности в окрестности $Q_{B r}=(2,0,0)$.

направлений соответствует одной ориентации антифазных доменов. Таким образом, для получения цепочек сателлитов в четырех направлениях $\langle 111\rangle$ необходимо существование антифазных доменов, ориентированных в четырех различных направлениях.

Расчеты интенсивности были также проведены вблизи основного брэгговского рефлекса. На рис. 6 приведены результаты расчетов одномерного профиля интенсивности и ее трехмерного распределения для $Q_{B r}=(2,0,0)$. Как следует из результатов моделирования, в окрестности $Q_{B r}$ не наблюдается каких-либо сателлитных рефлексов. Таким образом, в рамках рассматривае- мой модели не удается воспроизвести сателлитные рефлексы второго порядка в окрестности брэгговского рефлекса.

\section{4. Заключение}

В результате эксперимента по рассеянию синхротронного излучения на монокристалле ЦТС0.7 получена принципиально новая информация о структуре промежуточной фазы. Подтверждено, что в окрестности сверхструктурных рефлексов М-типа наблюдается семейство сателлитных рефлексов, положение которых 
может быть описано как $q_{M}+\{\delta, \delta, \delta\}$. Впервые установлено существование сателлитных рефлексов второго порядка вблизи сверхструктурных рефлексов М-типа в точках $q_{M}+\{2 \delta, 2 \delta, 2 \delta\}$ и в окрестности брэгговских рефлексов.

Было проведено моделирование картины рассеяния от антифазных доменов, внутри которых ионы свинца смещены антипараллельно в направлениях $\langle 110\rangle$. Показано, что картина рассеяния от такой регулярной системы доменов характеризуется наличием сверхструктурных рефлексов М-типа и сателлитных рефлексов первого и высших порядков в окрестности $Q_{M}$ точек. В рамках данной модели удалось достичь совпадения положений сателлитов относительно $Q_{M}$ точки в сравнении с экспериментально полученными результатами, а также достаточно хорошего воспроизведения относительных интенсивностей. В то же время в рамках данной модели не удалось воспроизвести сателлитные рефлексы второго порядка в окрестности брэгговских отражений.

Таким образом, существование сателлитов в окрестности основных рефлексов свидетельствует о необходимости пересмотреть предложенные механизмы возникновения сателлитов. Как показало моделирование, наличия антифазных доменов недостаточно для объяснения всей картины сверхструктурных отражений, наблюдаемых в промежуточной фазе. Можно предположить, что структура промежуточной фазы должна характеризоваться модуляцией, описываемой несоразмерным волновым вектором. С учетом большого количество сателлитных рефлексов выглядит вероятной необходимость введения нескольких таких векторов. В настоящее время на основании результатов исследования дифракции и диффузного рассеяния синхротронного излучения, полученных как в промежуточной, так и в параэлектрической фазах, ведется работа по построению модели, объясняющей структуру промежуточной фазы как результат несоразмерного фазового перехода.

\section{Финансирование работы}

Работа Д.А. Андрониковой поддержана стипендией Президента РФ SP-3762.2018.5 и Российским Фондом Фундаментальных Исследований (проект № 16-2914018). Работа А.В. Филимонова и Ю.А. Бронвальда выполнена при финансовой поддержке Российского Фонда Фундаментальных Исследований (проекты № 19-5253026 и 17-02-00774 соответственно). Д.Ю. Чернышов выражает благодарность за финансовую поддержку Министерству науки и высшего образования РФ в рамках Государственного задания, проект 3.1150.2017/4.6.

\section{Конфликт интересов}

Конфликт интересов отсутствует.

\section{Список литературы}

[1] G.H. Haertling. J. Am. Ceram. Soc. 82, 4, 797, (1999).

[2] A.K. Tagantsev, K. Vaideeswaran, S.B. Vakhrushev, A.V. Filimonov, R.G. Burk, A. Shaganov, D. Andronikova, A.I. Rudskoy, A.Q.R. Baron, H. Uchiyama. Nature Commun. 4, 3229, (2013)

[3] J. Hlinka, T. Ostapchuk, E. Buixaderas, C. Kadlec, P. Kuzel, I. Gregora, J.M. Savinov, A. Klic, J. Drahokoupil. Phys. Rev. Lett. 112, 19, 197601, (2014).

[4] J.Íñiguez, M. Stengel, S. Prosandeev, L. Bellaiche. Phys. Rev. B, 90, 22, 220 (2014).

[5] Z.G. Fthenakis, I. Ponomareva. Phys. Rev. B 96, 18, 184110, (2017).

[6] A.S. Mischenko, Q. Zhang, J.F. Scott, R.W. Whatmore, N.D. Mathur. Science 311, 5765, 1270 (2006).

[7] E. Glazkova-Swedberg, J. Cuozzo, S. Lisenkov, I. Ponomareva. Comp. Mater. Sci. 129, 44, (2017).

[8] H. Liu, B. Dkhil. Z. Kristallograph. Cryst. Mater. 226, 2, 163 (2011).

[9] X.-K. Wei, A.K. Tagantsev, A. Kvasov, K. Roleder, C.-L. Jia, N. Setter. Nature Commun. 5, 3031 (2014).

[10] S. Vakhrushev, D.A. Andronikova, D.Y. Chernyshov, A. Filimonov, S. Udovenk, N.V.R. Kumar. X-ray scattering by antiphase ferroelectric domain walls in antiferroelectric phase of the $\mathrm{PbZr}_{0.985} \mathrm{Ti}_{0.015} \mathrm{O}_{3}$. In Internet of Things, Smart and Next Generation Networks and Systems, 11118. Springer, (2018).

[11] Н.Г. Леонтьев, В.Г. Смотраков, Е.Г. Фесенко. Неорган. материалы 18, 3, 449 (1982).

[12] E. Sawaguchi, H. Maniwa, S. Hoshino. Phys. Rev. 83, 5, 1078, (1951).

[13] H. Fujishita, S. Hoshino. J. Phys. Soc. Jpn 53, 1, 226 (1984).

[14] A.M. Glazer, K. Roleder, J. Dec. Acta Crystallogr. B 49, 5 , 846, (1993).

[15] Y. Kuroiwa, Y. Terado, S.J. Kim, A. Sawada, Y. Yamamura, S. Aoyagi, E. Nishibori, M. Sakata, M. Takata. Jpn J. Appl. Phys. 44, 9S, 7151, (2005).

[16] D. Viehland. Phys. Rev. B 52, 2, 778, (1995).

[17] E. Buixaderas, T. Ostapchuk, J. Kroupa, M. Savinov, I. Gregora, J. Hlinka. Phase Transit. 87, 10-11, 1113, (2014).

[18] Z. Xu, X. Dai, J.-F. Li, D. Viehland. Appl. Phys. Lett. 66, 22, 2963, (1995)

[19] X. Dai, Z. Xu, D. Viehland. J. Am. Ceram. Soc. 78, 10, 2827, (1995).

[20] J. Ricote, D.L. Corker, R.W. Whatmore, S.A. Impey, A.M. Glazer, J. Dec, K.Roleder. J. Phys.: Condens. Matter 10, 8, 1767, (1998).

[21] S. Watanabe, Y. Koyama. Phys. Rev. B 63, 134103, (2001).

[22] S. Watanabe, Y. Koyama. Phys. Rev. B 66, 13, 134102, (2002).

[23] Z. Fu, X. Chen, P. Lu, C. Zhu, H. Nie, F. Xu, G. Wang, X. Dong. Cryst. Growth Des. 18, 8, 4395 (2018).

[24] A.K. Tagantsev, L.E. Cross, J. Fousek. Domains in ferroic crystals and thin films. Springer, (2010), $830 \mathrm{p}$.

[25] V. Dyadkin, P. Pattison, V. Dmitriev, D. Chernyshov. J. Synchrotron Rad. 23, 3, 825 (2016).

[26] Н.Г. Леонтьев, О.Е. Фесенко, В.Г. Смотраков. ФТТ 25, 7, 1958 (1983).

[27] ChrysAlis. Oxford Diffraction Ltd, Abingdon, England.

[28] A.M. Glazer. Acta Crystallographica A 31, 6, 756 (1975).

[29] Д.А. Андронникова. Фазовые переходы в чистом и допированном цирконате свинца. Дисс. канд. наук (2019).

[30] P.J. Brown. International tables for crystallography. V. C, Section 6.1. (2006). P. 554-590.

Редактор К.В. Емцев 